\title{
DHA mediates the protective effect of fish consumption on new episodes of depression among women
}

\author{
John L. Reeves ${ }^{1}$, Petr Otahal ${ }^{1}$, Costan G. Magnussen ${ }^{1,2}$, Terry Dwyer ${ }^{1,3}$, Antti J. Kangas ${ }^{4}$, Pasi Soininen ${ }^{4,5}$, \\ Mika Ala-Korpela ${ }^{4,5,6,7,8}$, Alison J. Venn ${ }^{1}$ and Kylie J. Smith ${ }^{1 *}$ \\ ${ }^{1}$ Menzies Institute for Medical Research, University of Tasmania, Private Bag 23, Hobart, Tasmania 7000, Australia \\ ${ }^{2}$ Research Centre of Applied and Preventive Cardiovascular Medicine, University of Turku, Turku 20014, Finland \\ ${ }^{3}$ George Institute for Global Health, University of Oxford, Oxford OX1 3QX, UK \\ ${ }^{4}$ Computational Medicine, Faculty of Medicine, University of Oulu, PO Box 5000, 90014 Oulu, Finland \\ ${ }^{5}$ NMR Metabolomics Laboratory, School of Pharmacy, University of Eastern, Finland, PO Box 1627, FI-70211 Kuopio, Finland \\ ${ }^{6}$ Population Health Science, Bristol Medical School, University of Bristol and Medical Research Council Integrative \\ Epidemiology Unit at the University of Bristol, Bristol BS8 2BN, UK \\ ${ }^{7}$ Systems Epidemiology, Baker Heart and Diabetes Institute, PO Box 6492, Melbourne, Victoria 3004, Australia \\ ${ }^{8}$ Department of Epidemiology and Preventive Medicine, School of Public Health and Preventive Medicine, Faculty of Medicine, \\ Nursing and Health Sciences, The Alfred Hospital, Monash University, 99 Commercial Road, Melbourne, Victoria 3004, Australia
}

(Submitted 5 October 2016 - Final revision received 4 September 2017 - Accepted 15 September 2017)

\section{Abstract}

In a longitudinal cohort study of young Australian adults, we reported that for women higher baseline levels of fish consumption were associated with reduced incidence of new depressive episodes during the 5-year follow-up. Fish are high in both $n$ - 3 fatty acids and tyrosine. In this study, we seek to determine whether $n$-3 fatty acids or tyrosine explain the observed association. During 2004-2006, a FFQ (nine fish items) was used to estimate weekly fish consumption among 546 women aged 26-36 years. A fasting blood sample was taken and highthroughput NMR spectroscopy was used to measure 233 metabolites, including serum $n-3$ fatty acids and tyrosine. During 2009-2011, new episodes of depression since baseline were identified using the lifetime version of the Composite International Diagnostic Interview. Relative risks were calculated using log-binomial regression and indirect effects estimated using the STATA binary_mediation command. Potential mediators were added to separate models, and mediation was quantified as the proportion of the total effect due to the mediator. The $n-3$ DHA mediated $25.3 \%$ of the association between fish consumption and depression when fish consumption was analysed as a continuous variable and $16.6 \%$ when dichotomised (reference group: $<2$ serves/week). Tyrosine did not mediate the association $(<0 \cdot 1 \%)$. Components in fish other than $n-3$ fatty acids and tyrosine might be beneficial for women's mental health.

Key words: Fish: Depression: $\boldsymbol{n}$-3 Fatty acids: Tyrosine: Young adults

Depression is a common cause of disability ${ }^{(1)}$. In the USA, depression cost an estimated $\$ 210 \cdot 5$ billion in 2010 and resulted in almost 400 million disability days during $2014^{(2)}$. There is increasing interest in whether lifestyle factors such as diet can prevent or treat depression ${ }^{(3)}$. A recent meta-analysis concluded that high fish consumption could reduce the risk of depres$\operatorname{sion}^{(4)}$. This raises the question of which component(s) of fish are responsible for the protective effect ${ }^{(5)}$.

One candidate is the $n-3$ PUFA, particularly the long-chain EPA and DHA, which are highly available in fish ${ }^{(6)}$, and DHA is highly concentrated in the brain ${ }^{(7)}$. The role of $n$-3 PUFA in neurophysiology and depression has been investigated extensively ${ }^{(8,9)}$, and there is evidence suggesting that long-chain $n-3$
PUFA may be beneficial in the prevention and treatment of depression $^{(9,10)}$, although the mechanism is debated $^{(8)}$ and there have not been enough randomised controlled trials with sufficient power to demonstrate this conclusively ${ }^{(11)}$. The ratio of $n$ - 6 PUFA to $n$ - 3 PUFA in erythrocytes has also been associated with depression in some studies ${ }^{(12)}$.

Another component of fish suggested to be beneficial for mental health is tyrosine ${ }^{(5)}$, an amino acid that is particularly abundant in some fish relative to other common forms of dietary protein ${ }^{(13)}$. Tyrosine is a precursor to dopamine, catecholamines, melanins and thyroid hormones ${ }^{(13)}$. Irregularities in these systems have been associated with neurophysiological and psychological problems, including depression ${ }^{(14-16)}$.

Abbreviations: CDAH, Childhood Determinants of Adult Health; DGI, dietary guideline index; DV, dependent variable; LNAA, large neutral amino acids. 
Acute tyrosine depletion in healthy volunteers has been linked to unipolar depression ${ }^{(17)}$, and chronic low levels of tyrosine in the brain have been associated with depression in longitudinal studies of phenylketonuria ${ }^{(18)}$. Tyrosine supplementation has been studied as a possible treatment for depression with mixed results ${ }^{(19)}$. There has only been one randomised double-blind trial; that study found that tyrosine supplementation was not an effective treatment for depression ${ }^{(20)}$

We previously reported that, among women, higher baseline fish consumption was associated with a lower risk of a new depressive episode during the 5-year follow-up, an association that remained robust when subjects with a history of depression were excluded ${ }^{(21)}$. Since publishing that paper, we have conducted metabolomics analysis on stored blood samples. This study investigates whether the protective association between fish consumption and depression observed among young Australian women is mediated by $n-3$ fatty acid status or tyrosine status.

\section{Methods}

\section{Study population}

The Childhood Determinants of Adult Health (CDAH) study is a follow-up to the 1985 Australian Schools Health and Fitness Survey, a nationally representative sample of 7- to 15-year-old Australian schoolchildren ( $n$ 8498). Participants were traced during 2001-2002 and 5170 enrolled in the CDAH study ${ }^{(22)}$. During 2004-2006, 2410 participants attended one of thirty-four study clinics held around Australia for physical measurements, aged 26-36 years (baseline for this analysis), and completed questionnaires. The majority of these participants (64\%) gave a fasting blood sample, and the characteristics of this subset are similar to the full set of participants (data not shown). In a second follow-up conducted 5 years later (2009-2011), participants completed written questionnaires and a telephone interview (follow-up).

The study was conducted according to the guidelines laid down in the Declaration of Helsinki, and all procedures involving human participants were approved by the Southern Tasmanian Health and Medical Ethics Committee. Written informed consent was obtained from all participants.

\section{Fish consumption}

Fish consumption was assessed at baseline via a 127-item $\mathrm{FFQ}^{(23)}$, with nine items relating to fish and seafood (canned fish, fresh fish, frozen fish, fried fish, mussels/oysters, lobster/ crayfish/yabbies (small lobsters), calamari/squid, prawns and other seafood $)^{(21)}$. The FFQ was based on a validated questionnaire developed for Australian adults ${ }^{(24,25)}$ and was a slightly modified version of the one used in the 1995 Australian National Nutrition Survey ${ }^{(26)}$. Participants were asked to recall how frequently they consumed each item over the previous 12 months by selecting one of nine response items, ranging from 'never/less than once a month' through to ' 6 or more times a day'. Weekly equivalents were calculated on the assumption that one serving was consumed on each occasion ${ }^{(23)}$, and then summed to determine the total weekly consumption of fish and seafood (hereafter 'weekly fish consumption'). Participants who did not answer all of the fish questions were excluded from the analysis.

\section{Depression}

At follow-up, participants completed the computerised lifetime version of the Composite International Diagnostic Interview (CIDI-Auto 2.1) via a computer-assisted telephone interview designed for use by non-clinical interviewers. Major depression or dysthymic disorder (hereafter 'depression') was defined by the Diagnostic and Statistical Manual of Mental Disorders, Fourth Edition (DSM-IV). Age at the time of the most recent episode was used to determine whether an episode had occurred since baseline. Participants who had a new episode of depression during the 5-year follow-up were compared with those who did not have a new episode.

\section{Metabolomics}

Fasting blood samples collected at baseline were stored at $-80^{\circ} \mathrm{C}$ for $11-13$ years before being analysed using the Computational Medicine metabolomic platform ${ }^{(27,28)}$, which used high-throughput NMR spectroscopy to profile the serum levels of 233 key metabolic markers. All samples were subjected to automated quality control, and values for any metabolic markers that could not be extracted reliably were excluded from the analysis ${ }^{(27,29)}$. Here we focus on tyrosine, DHA, total $n-3$ PUFA (including long-chain and short-chain $n-3$ PUFA) and total PUFA (including both $n-3$ and $n-6$ PUFA). We also examined the $n-3: n-6$ ratio, defined as (total $n-3$ PUFA)/(total $n-6$ PUFA), which includes both long- and SCFA. Although $n-6: n-3$ is the ratio more commonly used, we use the inverse form $(n-3: n-6)$ so that higher values are associated with greater protection. In addition, we examined the ratio of tyrosine:total concentration of large neutral amino acids (LNAA), as tyrosine competes with LNAA for transport across the blood-brain barrier ${ }^{(30)}$. LNAA included tyrosine, valine, leucine and isoleucine, but data for tryptophan were not available. The CV for this technique were $2 \cdot 7 \%$ for $n-3$ fatty acids $\left(2.7 \%\right.$ for DHA) and $7.7 \%$ for tyrosine ${ }^{(31)}$.

\section{Covariates}

At baseline, participants reported their marital status, education, occupation and smoking status. Weight and height were measured at study clinics using portable digital scales (Heine) and a portable stadiometer (Invicta). Height and weight were selfreported by participants who did not visit a study clinic and a correction factor was applied ${ }^{(32)}$. BMI was calculated as weight $(\mathrm{kg})$ divided by the square of height $\left(\mathrm{m}^{2}\right)$. Health status was assessed using the twelve-Item Short-Form Health Survey ${ }^{(33)}$. Information from the FFQ and food habits questionnaire was used to create a dietary guideline index (DGI), based on the Australian Guide to Healthy Eating ${ }^{(34)}$ and the Dietary Guidelines for Australian Adults ${ }^{(35)}$. The DGI included fifteen components, scored from 0 to 10 , with 10 indicating optimal intake (total range, 0-150). The DGI has been shown to be a valid measure of diet quality ${ }^{(36,37)}$. 


\section{Statistical analysis}

For each metabolic marker of interest, mediation was assessed using the four steps of mediation proposed by Baron \& Kenny ${ }^{(38)}$. This method tests potential mediators by examining the pathways from the independent variable to the mediator and from the mediator to the dependent variable, as well as considering the effect of controlling for the mediator on the pathway from the independent variable to the dependent variable. The four steps are as follows: (1) test whether the independent variable is associated with the dependent variable; (2) the independent variable is associated with the mediator; (3) the mediator variable is associated with the dependent variable; and (4) establish that the mediator variable mediates the association between the independent and dependent variables. In the first step, we tested the direct pathway between fish consumption (independent variable) and a new depressive episode (dependent variable) to verify the association established in our earlier study ${ }^{(21)}$. For the second step, we tested the individual associations between fish consumption (independent variable) and each metabolic marker (potential mediators), examining fish consumption as both a dichotomous and a continuous variable. For the third step, we tested the pathway from each metabolic marker (potential mediator) to depression (dependent variable), using log-binomial regression in each case. Finally, for the fourth step, we used the STATA binary_mediation command to estimate the indirect effects due to each potential mediator, and calculated this indirect effect as a proportion of the total effect. Internally, the binary_mediation command uses linear regression for continuous variables and (by default) logistic regression for categorical variables. Note that for the last three steps separate models were tested for each individual potential mediator.

For metabolites that passed all four mediation tests, we conducted further sensitivity analyses by including additional covariates in each model. Specifically, we included the same covariates as the earlier study: marital status, smoking status, weight status and self-rated health ${ }^{(21)}$. To determine whether diet quality was a confounder, we also included PUFA supplementation, the DGI score and a modified DGI score (which excluded fish items) in separate sensitivity analyses.

To determine whether the mediation effect of $n$ - 3 fatty acids and tyrosine differed by smoking status, we included a multiplicative interaction term between smoking status and fish intake. We found no evidence for interaction by smoking status, with all interactions terms $P>0 \cdot 33$.

This study focuses on women, as our earlier study found that fish consumption was not associated with depression among men. STATA software (2012, version 12.1; StataCorp) was used for all analyses.

\section{Results}

In total, 1552 women had data on baseline fish consumption. We excluded those who did not complete the CIDI-Auto 2.1 interview ( $n$ 627), were missing covariate data $(n 71)$, did not give a blood sample ( $n$ 304) or were missing values for tyrosine ( $n$ 4). The baseline characteristics of the 546 participants included in the analysis are shown in Table 1.
Table 1. Baseline characteristics among Australian women in the Childhood Determinants of Adult Health Study, stratified by depression status during the 5-year follow-up

(Numbers and percentages; mean values and standard deviations)

\begin{tabular}{|c|c|c|c|c|}
\hline \multirow[b]{3}{*}{ Characteristics } & \multirow{2}{*}{\multicolumn{2}{|c|}{$\frac{\text { No depression (n 448) }}{\text { Statistic } †}$}} & \multirow{2}{*}{\multicolumn{2}{|c|}{$\frac{\text { Depression* }^{*}(n \text { 98) }}{\text { Statistic } †}$}} \\
\hline & & & & \\
\hline & $n$ & $\%$ & $n$ & $\%$ \\
\hline \multicolumn{5}{|l|}{ Age (years) } \\
\hline Mean & \multicolumn{2}{|c|}{$31 \cdot 3$} & \multicolumn{2}{|c|}{$31 \cdot 1$} \\
\hline SD & \multicolumn{2}{|c|}{2.7} & \multicolumn{2}{|c|}{$2 \cdot 6$} \\
\hline Married or living as married & 333 & $74 \cdot 3$ & 63 & $64 \cdot 3$ \\
\hline Education & & & & \\
\hline University qualification & 251 & $56 \cdot 0$ & 47 & $48 \cdot 0$ \\
\hline Vocational & 95 & $21 \cdot 2$ & 16 & $16 \cdot 3$ \\
\hline High school or less & 102 & $22 \cdot 8$ & 35 & $35 \cdot 7$ \\
\hline \multicolumn{5}{|l|}{ Occupation } \\
\hline Professional or manager & 244 & $55 \cdot 2$ & 45 & $46 \cdot 4$ \\
\hline Non-manual & 101 & 22.9 & 30 & $30 \cdot 9$ \\
\hline Manual & 19 & $4 \cdot 3$ & 1 & 1.0 \\
\hline Not in the workforce & 78 & $17 \cdot 7$ & 21 & $21 \cdot 7$ \\
\hline \multicolumn{5}{|l|}{ BMI $\left(\mathrm{kg} / \mathrm{m}^{2}\right)$} \\
\hline Normal $(<25)$ & 293 & 65.4 & 60 & $61 \cdot 2$ \\
\hline Overweight (25-29.9) & 108 & $24 \cdot 1$ & 21 & 21.4 \\
\hline Obese $(\geq 30)$ & 47 & $10 \cdot 5$ & 17 & $17 \cdot 4$ \\
\hline \multicolumn{5}{|l|}{ Fish intake } \\
\hline$<2$ serves/week & 169 & $37 \cdot 7$ & 52 & $53 \cdot 1$ \\
\hline$\geq 2$ serves/week & 279 & $62 \cdot 3$ & 46 & 46.9 \\
\hline \multicolumn{5}{|l|}{ Serves/week } \\
\hline Mean & \multicolumn{2}{|c|}{$2 \cdot 81$} & \multicolumn{2}{|c|}{$2 \cdot 38$} \\
\hline SD & \multicolumn{2}{|c|}{$2 \cdot 21$} & \multicolumn{2}{|c|}{2.45} \\
\hline \multicolumn{5}{|l|}{ DHA (mmol/l) } \\
\hline Mean & \multicolumn{2}{|c|}{$0 \cdot 16$} & \multicolumn{2}{|c|}{0.15} \\
\hline SD & \multicolumn{2}{|c|}{0.06} & \multicolumn{2}{|c|}{0.05} \\
\hline \multicolumn{5}{|l|}{$n-3$ FA (mmol/l) } \\
\hline Mean & \multicolumn{2}{|c|}{0.38} & \multicolumn{2}{|c|}{0.36} \\
\hline SD & \multicolumn{2}{|c|}{$0 \cdot 10$} & \multicolumn{2}{|c|}{0.08} \\
\hline PUFA (mmol/l) & & & & \\
\hline Mean & & & & \\
\hline SD & & & & \\
\hline$n-3: n-6$ & & & & \\
\hline Mean & & & & \\
\hline SD & & & & \\
\hline Tyrosine $(\mathrm{mmol} / \mathrm{l})$ & & & & \\
\hline Mean & & & & \\
\hline SD & & & & \\
\hline
\end{tabular}

FA, fatty acids.

* New episode of depression in the preceding 5 years, as diagnosed by the Composite International Diagnostic Interview (Composite International Diagnostic InterviewAuto 2.1).

† Sample sizes vary owing to missing data (range 442-448 for women without depression; 97-98 for women who had a depressive episode).

In total, ninety-eight women had a depressive episode during the 5-year follow-up. Although the study population was smaller than our previous study ${ }^{(21)}$, as not all participants gave a blood sample, a similar association between fish consumption and depression was found (7.6\% lower risk of a new depressive episode with every additional serve of fish), verifying Step 1 of the Baron and Kenny mediation tests.

The results for the three remaining tests are summarised in Table 2 in descending order of mediation, for the key metabolic markers of interest, and in the online Supplementary Tables for all metabolites. DHA was the only metabolic marker to pass all three tests. DHA levels were positively associated with fish consumption (continuous: $\beta=0.005, P<0.001$; dichotomous: 
Table 2. The mediation effect of $n-3$ fatty acids and tyrosine on the association between fish consumption at baseline and new episodes of depression in the 5-year follow-up

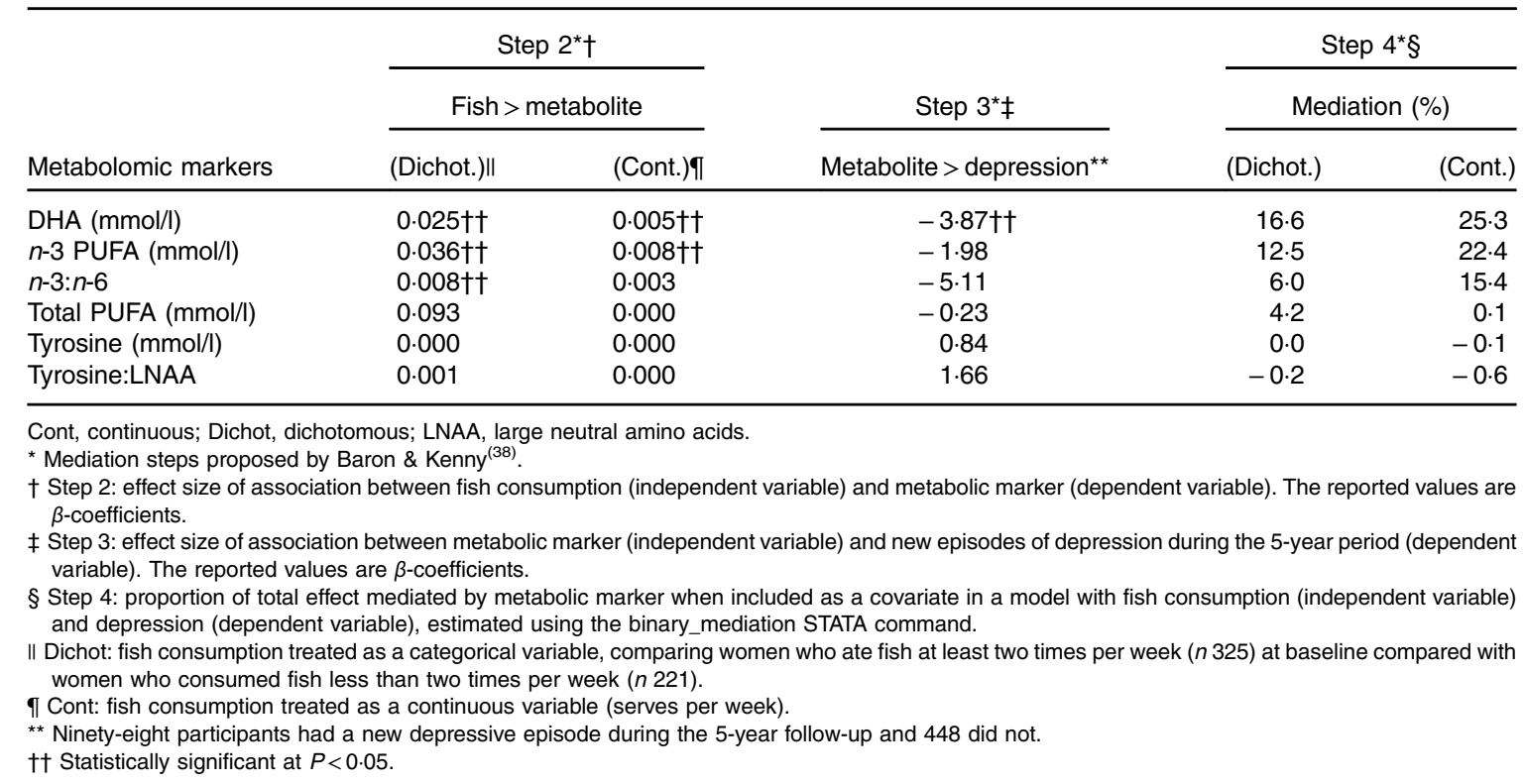

$\beta=0 \cdot 025, P<0 \cdot 001$, Table 2$)$ and negatively associated with depression $(\beta=-3 \cdot 87, P=0 \cdot 03)$. As a proportion of the total effect, the indirect effect due to DHA was the highest of all metabolites examined, estimated at $25 \%$ (continuous) and $17 \%$ (dichotomous). The associations remained in the sensitivity analyses when covariates were added to the models.

Tyrosine levels and the tyrosine:LNAA ratio were also positively associated with fish consumption, although the effect sizes were very small and NS $(\beta<0.001, P=0.83$ for tyrosine and $\beta<0.001$, $P=0.57$ for tyrosine:LNAA, Table 2 ). However, rather than being protective against depression, higher levels of tyrosine and the tyrosine:LNAA ratio were associated with a higher risk of depression, but again these associations were not significant ( $\beta=0.84, P=0.92$ for tyrosine and $\beta=1.66, P=0.75$ for tyrosine: LNAA). These associations were not sustained during sensitivity testing when PUFA supplementation and the covariates from the original study were included in the model. Tyrosine (but not tyrosine:LNAA) passed all three mediation tests, but the proportion of the total effect mediated by tyrosine was $<1 \%$.

\section{Discussion}

This study builds on our previous work that reported higher baseline fish consumption was associated with a lower risk of a new depressive episode among young women ${ }^{(21)}$. In this study, we have shown that the association is partially mediated by baseline plasma levels of DHA but not tyrosine.

DHA only partially explained the protective role of fish against depression, suggesting that there may be other components in fish that are protective or there may be synergistic effects between the nutrients in fish. The mediation proportion for DHA was roughly three to four times higher than the highest non- $n$ - 3 fatty acid-related metabolites. DHA passed all three steps of the Baron and Kenny method for all models tested, including models with smoking status and use of fish oil supplements as covariates. We did not adjust for age or physical activity, as these variables were not associated with depression in this sample and therefore cannot be confounders ${ }^{(21)}$, but other unmeasured confounding is possible.

In the current study, total $n-3$ PUFA and the $n-3: n-6$ ratio did not satisfy all criteria for mediation. The mediation effect for $n-3$ PUFA was lower than that of DHA alone, probably because the effect of DHA is diluted by the inclusion of $n-3$ PUFA that do not have a protective effect against depression, such as $\alpha$-linolenic acid.

To our knowledge, no previous studies have conducted mediation analysis to identify the components in fish that mediate the protective effect of fish on depression. Although we do not have a measure for EPA in our study, our findings add to the previous literature examining associations between plasma/ serum or dietary $n-3$ fatty acids and depression. A meta-analysis of fourteen studies found that DHA, EPA and $n-3$ fatty acid levels in plasma/serum were lower among patients with depression than controls ${ }^{(4)}$. A cross-sectional study in Japanese adults (40+ years) found an inverse association between serum DHA and EPA levels and depressive symptoms ${ }^{(39)}$. Dietary intake of DHA and EPA was found to be inversely associated with the risk of depression symptoms after a 3-year follow-up in the Coronary Artery Risk Development in Young Adults (CARDIA) study ${ }^{(40)}$. In contrast, several longitudinal studies have reported no association between dietary intake of $n$-3 PUFA and depression ${ }^{(41-43)}$. The conflicting results may reflect the different methods used to assess fish intake and depression, different ages of the samples (e.g. older adults or having a wide age range) and unmeasured or residual confounding.

$n$-3 Fatty acid supplements have been tested in randomised controlled trials as a possible treatment of depression, with inconsistent results. Two recent meta-analyses published in 2016 have conflicting conclusions. A Cochrane systematic 
review and meta-analysis of twenty randomised controlled trials found insufficient evidence to support the use of $n$ - 3 fatty acid supplements as a treatment for depression ${ }^{(11)}$. Most studies included in the review were small (all but three studies had $<100$ participants), and of short duration (4-16 weeks). In contrast, a meta-analysis of thirteen studies found a beneficial effect of $n-3$ fatty acid supplementation in patients with depression, especially for higher doses of EPA and when used in combination with antidepressants ${ }^{(44)}$. However, the beneficial effect was small, and it has been argued that the effect may not be clinically important and the results were influenced by poorerquality studies $^{(45)}$.

There are several limitations that need to be considered. The FFQ did not distinguish between fatty and non-fatty fish, and it is possible that analysis focusing on fatty fish may reveal a stronger association with depression, as well as a higher degree of mediation by $n$-3 PUFA. Data for one LNAA (tryptophan) were not available, and therefore we overestimate the tyrosine:LNAA ratio. There is the possibility that some metabolites may have degraded as a result of long-term storage, but samples were subjected to quality control. Of the 1940 participants who had blood samples analysed from the CDAH study, seventeen (0.8\%) were excluded from the analysis for degraded $n-3$ fatty acids (eight women), and twenty-seven (1.4\%) for tyrosine (nine women). Compounds representing lipid oxidation products were not detected in any of the samples.

Another limitation was the difference in time scales between the variables being compared. Although the FFQ assesses fish consumption retrospectively over a 12-month period and depression status was determined over a 5-year period, the metabolomic data represent a snapshot of fasting plasma levels at a single moment in time. This snapshot reflects dietary intake over the previous few days, but different nutrients are metabolised at different rates and so the degree to which fasting plasma metabolite levels can be taken as a proxy for long-term consumption may vary. Plasma and erythrocytes $n$-3 PUFA levels have both been shown to correlate to short-term consumption ${ }^{(46)}$. However, tyrosine is rapidly metabolised in the body, with levels returning to baseline within a few hours after a meal ${ }^{(47)}$. Long-term consumption patterns are difficult to capture owing to tyrosine's multiple destinations in the body, although future studies with more frequent measurements may provide a more accurate picture. Long-term fish intake appears to be relatively stable in this cohort, as sensitivity analyses in our previous study found similar results when baseline and follow-up intake was averaged and used as the exposure variable ${ }^{(21)}$. Although it would have been ideal to also examine EPA, DHA was the only long-chain n-3 PUFA for which individual levels were available.

Data on lipid mediators or metabolites derived from PUFA may help to clarify the role of PUFA in the association between fish consumption and depression. However, EPA and lipid mediators are unable to be distinguished by the NMR platform, and complementary assays such as MS may be required. In addition, we were unable to examine change in metabolites as blood samples, and therefore the metabolite data were only collected at baseline. The study population had higher representation of professionals/managers and people married or living as married than the general Australian population of similar age. Although marital status or socio-economic status may play a role in determining susceptibility of depression, these factors are unlikely to affect how serum metabolites mediate the association between diet and depression.

Major strengths of this study include the ability to compare 233 potential mediators in the same sample using a rigorous and accepted method of mediation. Depression was assessed using a diagnostic tool, considered the 'gold standard' in epidemiological studies.

These findings add to a growing body of evidence that suggests that DHA may have a protective role for mental health. DHA only partially explained the protective association between fish consumption and depression, suggesting that other components in fish may benefit women's mental health.

\section{Acknowledgements}

The authors gratefully acknowledge the contribution of Marita Dalton (study project manager), all other project staff and the study participants.

This project was funded by grants from the National Health and Medical Research Council (grants 211316, 544923); the National Heart Foundation (GOOH0578); the Select Foundation (M0020771); the Tasmanian Community Fund (D0013808); and Veolia Environmental Services. C. G. M. was supported by a National Heart Foundation of Australia Future Leader Fellowship (100849); M. A.-K. by the Sigrid Juselius Foundation and the Strategic Research Funding from the University of Oulu, Finland; A. J. V. by a National Health and Medical Research Council Fellowship (APP1008299); and K. J. S. by a National Health and Medical Research Council Early Career Fellowship (APP1072516). M. A.-K. works in a Unit that is supported by the University of Bristol and UK Medical Research Council (MC_UU_12013/1). The study was sponsored by Sanitarium (Melbourne, Victoria, Australia), ASICS Ltd (Kobe, Japan) and Target Australia Pty Ltd (North Geelong, Victoria, Australia). The funding bodies had no role in the study design, analysis or writing of this article.

K. J. S., A. J. V. and T. D. designed the research. J. L. R. performed the statistical analysis with support from K. J. S., P. O. and C. G. M.. A. J. K., P. S. and M. A.-K. analysed the metabolomics data. J. L. R. and K. J. S. wrote the paper with critical input from C. G. M., P. O. and A. J. V. All authors provided critical revision of the manuscript and approved the final version.

A. J. K. and P. S. are shareholders of Nightingale Health (former Brainshake Ltd), a company offering NMR-based metabolite profiling. The other authors declare that there are no conflicts of interest.

\section{Supplementary material}

For supplementary material/s referred to in this article, please visit https://doi.org/10.1017/S0007114517002768

\section{References}

1. Vos T, Flaxman AD, Naghavi M, et al. (2012) Years lived with disability (YLDs) for 1160 sequelae of 289 diseases and injuries 1990-2010: a systematic analysis for the Global Burden of Disease Study 2010. Lancet 380, 2163-2196. 
2. Greenberg PE, Fournier A-A, Sisitsky T, et al. (2015) The economic burden of adults with major depressive disorder in the United States (2005 and 2010). J Clin Psychiatry 76, 155-162.

3. Sarris J, O'Neil A, Coulson CE, et al. (2014) Lifestyle medicine for depression. BMC Psychiatry 14, 107.

4. Li F, Liu X \& Zhang D (2016) Fish consumption and risk of depression: a meta-analysis. J Epidemiol Community Health 70, 299-304.

5. Evans JM (2014) Re: 'Longitudinal associations between fish consumption and depression in young adults'. Am J Epidemiol $180,557$.

6. Nichols PD, Petrie J \& Singh S (2010) Long-chain omega-3 oilsan update on sustainable sources. Nutrients 2, 572-585.

7. Youdim KA, Martin A \& Joseph JA (2000) Essential fatty acids and the brain: possible health implications. Int J Dev Neurosci 18, 383-399.

8. Mello AH, Gassenferth A, Souza LR, et al. (2014) Omega-3 and major depression: a review. Acta Neuropsychiatr 26, 178-185.

9. Deacon G, Kettle C, Hayes D, et al. (2017) Omega 3 polyunsaturated fatty acids and the treatment of depression. Crit Rev Food Sci Nutr 57, 212-223.

10. Appleton KM, Rogers PJ \& Ness AR (2010) Updated systematic review and meta-analysis of the effects of $n-3$ long-chain polyunsaturated fatty acids on depressed mood. Am J Clin Nutr 91, 757-770.

11. Appleton KM, Sallis HM, Perry R, et al. (2016) $\omega-3$ Fatty acids for major depressive disorder in adults: an abridged Cochrane review. BMJ Open 6, e010172.

12. Sontrop J \& Campbell MK (2006) $\omega-3$ Polyunsaturated fatty acids and depression: a review of the evidence and a methodological critique. Prev Med 42, 4-13.

13. Tyrrell HA (1998) Tyrosine: food supplement or therapeutic agent? J Nutr Environ Med 8, 349-359.

14. Dailly E, Chenu F, Renard CE, et al. (2004) Dopamine, depression and antidepressants. Fundam Clin Pharmacol 18, 601-607.

15. Anand A \& Charney DS (2000) Norepinephrine dysfunction in depression. J Clin Psychiatry 61, 16-24.

16. Jackson IM. (1998) The thyroid axis and depression. Thyroid 8, 951-956.

17. McLean A, Rubinsztein JS, Robbins TW, et al. (2004) The effects of tyrosine depletion in normal healthy volunteers: implications for unipolar depression. Psychopharmacology (Berl) 171, 286-297.

18. Clacy A, Sharman R \& McGill J. (2014) Depression, anxiety, and stress in young adults with phenylketonuria: associations with biochemistry. J Dev Behav Pediatr 35, 388-391.

19. Gelenberg AJ \& Gibson CJ (1984) Tyrosine for the treatment of depression. Nutr Health 3, 163-173.

20. Gelenberg AJ, Wojcik JD, Falk WE, et al. (1990) Tyrosine for depression: a double-blind trial. J Affect Disord 19, $125-132$

21. Smith KJ, Sanderson K, McNaughton SA, et al. (2014) Longitudinal associations between fish consumption and depression in young adults. Am J Epidemiol 179, 1228-1235.

22. Gall SL, Jose K, Smith K, et al. (2009) The Childhood Determinants of Adult Health Study: a profile of a cohort study to examine the childhood influences on adult cardiovascular health. Australas Epidemiol 16, 35-38.

23. Smith KJ, McNaughton SA, Gall SL, et al. (2009) Takeaway food consumption and its associations with diet quality and abdominal obesity: a cross-sectional study of young adults. Int J Behav Nutr Phys Act 6, 29.

24. Ireland P, Jolley D, Giles G, et al. (1994) Development of the Melbourne FFQ: a food frequency questionnaire for use in an Australian prospective study involving an ethnically diverse cohort. Asia Pac J Clin Nutr 3, 19-31.
25. Hodge A, Patterson AJ, Brown WJ, et al. (2000) The Anti Cancer Council of Victoria FFQ: relative validity of nutrient intakes compared with weighed food records in young to middle-aged women in a study of iron supplementation. Aust N Z J Public Health 24, 576-583.

26. McLennan W \& Podger AS (1998) National Nutrition Survey Users' Guide, 1995. Canberra: Australian Bureau of Statistics \& Commonwealth Department of Health and Family Services.

27. Würtz P, Kangas AJ, Soininen P, et al. (2017) Quantitative serum nuclear magnetic resonance metabolomics in largescale epidemiology a primer on -omic technology. Am J Epidemiol (epublication ahead of print version 16 September 2017).

28. Soininen P, Kangas AJ, Wurtz P, et al. (2009) High-throughput serum NMR metabonomics for cost-effective holistic studies on systemic metabolism. Analyst 134, 1781-1785.

29. Soininen P, Kangas AJ, Wurtz P, et al. (2015) Quantitative serum nuclear magnetic resonance metabolomics in cardiovascular epidemiology and genetics. Circ Cardiovasc Genet 8, 192-206.

30. Demling J, Langer K, Wörthmüller M, et al. (1993) Individual day-to-day variations in plasma amino acid levels in healthy persons. Amino Acids 5, 253-262.

31. Kettunen J, Demirkan A, Wurtz P, et al. (2016) Genome-wide study for circulating metabolites identifies 62 loci and reveals novel systemic effects of LPA. Nat Commun 7, 11122.

32. Venn AJ, Thomson RJ, Schmidt MD, et al. (2007) Overweight and obesity from childhood to adulthood: a follow-up of participants in the 1985 Australian Schools Health and Fitness Survey. Med J Aust 186, 458.

33. Ware JE Jr, Kosinski M \& Keller SD (1996) A 12-Item ShortForm Health Survey: construction of scales and preliminary tests of reliability and validity. Med Care 34, 220-233.

34. Smith A, Schmerlaib Y \& Kellett E (1998) The Australian Guide to Healthy Eating: Background Information for Nutrition Educators. Canberra: Commonwealth Department of Health and Family Services.

35. National Health and Medical Research Council (2003) Dietary Guidelines for Australian Adults. Canberra: National Health and Medical Research Council.

36. McNaughton SA, Ball K, Crawford D, et al. (2008) An index of diet and eating patterns is a valid measure of diet quality in an Australian population. J Nutr 138, 86-93.

37. McNaughton SA, Dunstan DW, Ball K, et al. (2009) Dietary quality is associated with diabetes and cardio-metabolic risk factors. J Nutr 139, 734-742.

38. Baron RM \& Kenny DA (1986) The moderator-mediator variable distinction in social psychological research: conceptual, strategic, and statistical considerations. J Pers Soc Psychol 51, 1173-1182.

39. Horikawa C, Otsuka R, Kato Y, et al. (2016) Cross-sectional association between serum concentrations of $n$-3 long-chain PUFA and depressive symptoms: results in Japanese community dwellers. Br J Nutr 115, 672-680.

40. Colangelo LA, He K, Whooley MA, et al. (2009) Higher dietary intake of long-chain omega-3 polyunsaturated fatty acids is inversely associated with depressive symptoms in women. Nutrition 25, 1011-1019.

41. Hakkarainen R, Partonen T, Haukka J, et al. (2004) Is low dietary intake of omega-3 fatty acids associated with depression? Am J Psychiatry 161, 567-569.

42. Jacka FN, Pasco JA, Henry MJ, et al. (2004) Dietary omega-3 fatty acids and depression in a community sample. Nutr Neurosci 7, 101-106.

43. Lucas M, Mirzaei F, O'Reilly EJ, et al. (2011) Dietary intake of $n-3$ and $n-6$ fatty acids and the risk of clinical depression in women: a 10-y prospective follow-up study. Am J Clin Nutr $\mathbf{9 3}$, $1337-1343$. 
44. Mocking RJ, Harmsen I, Assies J, et al. (2016) Meta-analysis and meta-regression of omega-3 polyunsaturated fatty acid supplementation for major depressive disorder. Transl Psychiatry 6, e756.

45. Bastiaansen JA, Munafo MR, Appleton KM, et al. (2016) The efficacy of fish oil supplements in the treatment of depression: food for thought. Transl Psychiatry 6, e975.
46. Witte TR, Salazar AJ, Ballester OF, et al. (2010) RBC and WBC fatty acid composition following consumption of an omega 3 supplement: lessons for future clinical trials. Lipids Health Dis 9, 1.

47. Milsom JP, Morgan MY \& Sherlock S (1979) Factors affecting plasma amino acid concentrations in control subjects. Metabolism 28, 313-319. 